\title{
Perturbations apportées aux caractéristiques de fonctionnement des turbomachines hydrauliques par des écarts de similitude géométrique
}

\author{
Upsetting effects of geometrical simillarity errors \\ on the operating characteristics of hydraulic turbomachines
}

Richard Canavelis

Bergeron S.A.

\author{
Robert Bonnafoux
}

EDF - Direction des Études

et Recherches (Chatou)

\author{
Robert Philibert
Société Neyrpic \\ Robert $\mathbb{P h i l i b e r t}$
Société Neyrpic
}

Rémy Simon

EDF - Septen

\section{Introduction}

Les caractéristiques de fonctionnement d'une turbomachine hydraulique, telles que déterminées à partir des essais de réception de la machine industrielle, peuvent différer plus ou moins des caractéristiques prévisionnelles établies initialement au niveau du projet. Une cause essentielle de ces écarts de fonctionnement réside dans l'existence obligatoire d'écarts de similitude géométrique entre la machine industrielle d'une part et les plans de tracé hydraulique ou le modèle réduit d'autre part.

Nous tenterons de montrer dans ce qui suit l'importance que peuvent revêtir dans la pratique ces écarts de similitude géométrique en indiquant quelques formules simplifiées qui permettent d'estimer les effets de tels écarts, et en présentant quelques cas particuliers de réalisations industrielles pour lesquelles une analyse de ces écarts a été effectuée. Nous présenterons enfin quelques remarques sur la compatibilité des incertitudes de fabrication avec les tolérances contractuelles imposées au constructeur.

Estimation des perturbations apportées par les écarts de similitude géométrique.

Incertitudes entourant les caractéristiques de fonctionnement d'une turbomachine hydraulique

Les caractéristiques de fonctionnement d'une turbomachine hydraulique peuvent être établies à différents stades du déroulement d'un projet ou d'une affaire.

Au moment du projet, le constructeur établit des caractéristiques prévisionnelles de la machine indus- trielle. Pour ce faire, il utilise souvent les résultats d'essai d'un modèle réduit. L'incertitude qui entoure les caractéristiques prévisionnelles provient essentiellement des incertitudes élémentaires suivantes :

- Incertitudes relatives aux mesures des grandeurs hydrauliques, mécaniques ou électriques durant les essais du modèle réduit.

- Incertitudes sur les effets d'échelle hydrauliques provenant d'écarts de similitude des écoulements entre le modèle réduit et la machine industrielle.

- Incertitudes de fabrication entraînant des écarts de similitude géométrique entre modèle réduit et machine industrielle.

Il convient de noter que les écarts de similitude géométrique entre modèle réduit et machine industrielle peuvent être de deux natures différentes.

Certains écarts sont systématiques et correspondent à des écarts de cote ou de forme connus en moyenne à l'avance et souvent imposés par des procédés de fabrication ou des exigences mécaniques ; épaisseurs d'aubages, états de surface, jeux aux labyrinthes. Ces écarts conduisent plus à un déport de caractéristiques qu'à une incertitude. Ils introduisent toutefois une légère incertitude dans la mesure où leurs effets ne peuvent être qu'estimés.

Certains écarts sont au contraire totalement involontaires et aléatoires parce que liés aux aléas de fabrication : aléas de fonderie, précision d'usinage etc... Ces écarts conduisent à une incertitude totale qui ne peut être levée qu'en effectuant un contrôle dimensionnel de la machine.

Le contrôle dimensionnel tant du modèle réduit que de la machine industrielle permet en principe de réduire grandement les incertitudes de mesure géométrique. Le contrôle du modèle réduit précède en général l'établis- 
sement des caractéristiques prévisionnelles initiales. Il n'en est pas de même pour la machine industrielle qui ne peut être contrôlée dimensionnellement qu'après sa réalisation complète. Ce n'est qu'à ce stade, que l'on peut établir des caractéristiques prévisionnelles corrigées après contrôle dimensionnel.

$\mathrm{Au}$ moment des essais de la machine industrielle en laboratoire ou sur le site, on établit enfin des caractéristiques expérimentales qui sont elles mêmes entourées des incertitudes relatives aux mesures de grandeurs hydrauliques, mécaniques ou électriques imputables aux conditions d'essai.

Il apparaît ainsi qu'entre une caractéristique expérimentale déduite d'essais sur modèle réduit et la caractéristique correspondante déduite des essais de la machine industrielle, le constructeur doit estimer les écarts maximaux à craindre en tenant compte de la somme de toutes les incertitudes mentionnées ci-dessus. On est donc amené, au niveau du projet, à établir des courbes caractéristiques moyennes probables, entourées d'une bande d'incertitude correspondant à un niveau de probabilité donné (qui est en général de $95 \%$ ). Ce n'est, en toute rigueur, qu'à partir de ces éléments, que l'on peut fixer les garanties techniques d'un contrat.

\section{Analyse des effets de quelques écarts de similitude géométrique}

Nous nous limiterons, dans ce qui suit, au cas des pompes hydrauliques ou à celui de la marche en pompe des pompes-turbines. Nous considérerons, par ailleurs, exclusivement la zone de rendement maximal, étant donné qu'en dehors de cette zone, il est difficile de rattacher la forme des caractéristiques de fonctionnement à des lois générales bien établies. Enfin, dans un but de simplification, nous n'évoquerons que quelques paramètres géométriques essentiels des roues centrifuges (ou hélico-centrifuges), dont l'influence s'avère assez facilement quantifiable à l'aide de formules simples.

Nous supposerons en particulier que les écarts de similitude géométrique considérés sont suffisamment faibles pour que la caractéristique adimensionnelle de la hauteur interne de la roue reste inchangée entre le modèle réduit et la pompe industrielle. Dans ces conditions, cette caractéristique est représentée au voisinage du rendement maximal par une droite d'équation :

$$
\frac{V u_{2}}{U_{2}}=A-B \frac{V e_{2}}{U_{2}} \operatorname{cotg} \beta_{2}
$$

$U_{2} \quad$ Vitesse périphérique de rotation à la sortie de la roue

$V u_{2}$ Composante tangentielle de la vitesse absolue à la sortie de la roue

$\mathrm{Ve}_{2}$ Composante radiale de la vitesse absolue à la sortie de la roue

$\beta_{2} \quad$ Angle moyen de sortie des aubes de la roue

$A, B$ Constantes expérimentales adimentionnelles qui dépendent entre autres du nombre d'aubes $Z_{R}$ de la roue et du style du tracé hydraulique.

Dans le plan $Q_{R}$ (débit délivré par la roue), $H i$ (hauteur interne de la roue) - l'équation (1) devient :

$$
H i=A \frac{U_{2}^{2}}{g}-\frac{B U_{2} \operatorname{cotg} \beta_{2}}{D_{2} b_{2} g} \cdot Q_{R}
$$

$b_{2}$ Largeur de sortie des canaux de la roue

$D_{2}$ Diamètre de sortie des aubes de la roue

Influence de la variation de la largeur de sortie $b_{2}$ des canaux de la roue

Le débit $Q_{R}$ délivré par la roue pour une hauteur interne $H i$ est donné par la relation :

$$
Q_{R}=\pi D_{2} \cdot b_{2} \cdot V e_{2}
$$

En supposant le rendement hydraulique $\eta_{H}=H / H i$ et le rendement volumétrique $\eta_{v}=Q / Q_{R}$ quasiment inchangés, on voit aisément que la variation relative du débit utile $Q$ à hauteur énergétique totale $H$ constante est donnée par :

$$
\left(\frac{\Delta Q}{Q}\right)_{b_{2}}=\frac{\Delta b_{2}}{b_{2}}
$$

De même, pour le nouveau point de fonctionnement $Q+(\Delta Q)_{b_{2}}, H$. la puissance absorbée subit une variation relative donnée en première approximation par:

$$
\left(\frac{\Delta P}{P}\right)_{b_{2}}=\left(\frac{\Delta Q}{Q}\right)_{b_{2}}
$$

Sauf dispositions particulières, les roues industrielles sortent de fonderie en présentant des écarts relatifs de largeur pouvant atteindre $\pm 2 \%$ dans de nombreux cas. Ces écarts entraînent donc une incertitude relative identique $(\Delta Q / Q)_{b_{2}}$ et $(\Delta P / P)_{b_{2}}$ sur la position des caractéristiques hauteur-débit et puissance-débit.

Influence de la variation de l'angle de sortie des aubes $\beta_{2}$

En supposant le rendement volumétrique et le rendement hydraulique quasiment inchangés, on voit aisément en dérivant la relation (2), que la variation relative de débit $Q$ à hauteur énergétique totale $H$ donnée, est égal à :

$$
\left(\frac{\Delta Q}{Q}\right)_{\beta_{2}}=\frac{\Delta \beta_{2}}{\sin \beta_{2} \cdot \cos \beta_{2}}\left(\beta_{2} \text { en radian }\right)
$$

Pour le nouveau point de fonctionnement $Q+(\Delta Q)_{\beta_{2}}, H$, la puissance absorbée subit une variation relative donnée en première approximation par :

$$
\left(\frac{\Delta P}{P}\right)_{\beta_{2}}=\left(\frac{\Delta Q}{Q}\right)_{\beta_{2}}
$$

Les incertitudes de fonderie peuvent conduire dans de nombreux cas à des écarts angulaires d'environ $\pm 1^{\circ}$.

Toutefois, dans le cas de fabrications particulièrement soignées, on peut estimer ces incertitudes ramenées à une valeur inférieure à $\pm 30^{\prime}$. II convient néanmoins de noter que la valeur moyenne de l'angle $\beta_{2}$ sur une certaine longueur de la sortie des aubes s'avère difficile à apprécier avec une définition meilleure que $15^{\prime}$.

Notons que pour un angle $\beta_{2}$ voisin de $20^{\circ}$, une incertitude $\Delta \beta_{2}= \pm 30^{\prime}$ conduit à une incertitude de $(\Delta Q / Q)_{\beta_{2}}= \pm 2,7 \%$ 
Influence de la variation de l'épaisseur moyenne de sortie des aubes $e_{2}$

Toute variation d'épaisseur des aubes modifie pour un angle $\beta_{2}$ donné, l'aire de la section de sortie des canaux et par suite la vitesse moyenne relative dans cette section.

Pour une valeur donnée de la vitesse $V e_{2}$, on peut supposer, en première approximation le débit $Q_{R}$ proportionnel à l'ouverture moyenne $a_{2}$ de sortie des canaux.

Or

$$
a_{2} \# \frac{\pi D_{2}}{Z_{R}} \sin \beta_{2}-K \times e_{2}
$$

En supposant le rendement volumétrique et le rendement hydraulique quasiment inchangés, la variation relative du débit $Q$ à hauteur énergétique totale $H$ donnée est alors égale à la variation relative $\Delta a_{2} / a_{2}$ soit :

$$
\left(\frac{\Delta Q}{Q}\right)_{e_{2}}=-\frac{K}{\frac{\pi D_{2} \sin \beta_{2}-K}{Z_{R} \cdot e_{2}}} \frac{\Delta e_{2}}{e_{2}}
$$

$K$ est un paramètre de forme qui dépend de l'affûtage de sortie des aubes et qui prend les valeurs suivantes:

- affûtage nul $K=1$

- affûtage entièrement sur l'avant $\quad K=1$

- affûtage moitié avant, moitié arrière $K=0,5$

- affûtage entièrement sur l'arrière $\quad K=0$

Pour le nouveau point de fonctionnement $Q+(\Delta Q)_{e}, H$, la puissance absorbée subit une variation relative donnée en première approximation par :

$$
\left(\frac{\Delta P}{P}\right)_{e_{2}}=\left(\frac{\Delta Q}{Q}\right)_{e_{2}}
$$

Les incertitudes de fonderie peuvent souvent conduire à une incertitude $\Delta e_{2} / e_{2}$ approchant $\pm 20 \%$. Dans ces conditions, à titre d'exemple, une roue correspondant à $\beta_{2}=20^{\circ}, D_{2}=0,8 \mathrm{~m}, Z_{R}=6, e_{2}=10 \mathrm{~mm}$ conduirait à une incertitude $(\Delta Q / Q)_{e_{2}}$ pouvant atteindre $\pm 1,5 \%$.

Par ailleurs, il arrive fréquemment qu'un modèle réduit réalisé en fonderie sorte avec des aubes proportionnellement plus épaisses que celles de la machine industrielle. Ainsi, un modèle de $350 \mathrm{~mm}$ de diamètre extérieur avec des aubes de $5 \mathrm{~mm}$ d'épaisseur peut donner lieu à une pompe industrielle de $3,5 \mathrm{~m}$ de diamètre extérieur dont les aubes n'auraient qu'une épaisseur voisine de $25 \mathrm{~mm}$, ce qui se traduit par une diminution relative d'épaisseur $\Delta e_{2} / e_{2}=-50 \%$. Une telle diminution peut se traduire par un gain en débit $(\triangle Q f Q)_{e_{2}}$ de plusieurs pour cent.

\section{Influence de la variation de l'angle d'entrée des aubes $\beta_{1}$}

Toute modification de l'aire de la section d'entrée des canaux d'une roue modifie la vitesse relative moyenne dans cette section pour un débit donné. Or on peut estimer que les caractéristiques de NPSH correspondant à des débits supérieurs au débit d'adaptation de l'œillard sont essentiellement fonction de la vitesse moyenne dans cette section d'entrée, la cavitation se produisant sur la face cachée de l'entrée des aubes.

L'aire de la section d'entrée peut être considérée en première approximation comme proportionnelle à l'ouverture $a_{1}$ de l'entrée du canal sur un diamètre $D_{1}$ de référence avec:

$$
a_{1} \# \frac{\pi D_{1}}{Z_{R}} \sin \beta_{1}-K^{\prime} e_{1}
$$

$e_{1}$ épaisseur moyenne des aubes à l'entrée de la roue $K^{\prime}$ paramètre d'affûtage prenant les valeurs suivantes :
- affûtage nul
$K^{\prime}=1$
- affûtage entièrement sur l'arrière
$K^{\prime}=1$
- affûtage moitié avant, moitié arrière
- affûtage entièrement sur l'avant
$K^{\prime}=0,5$
$K^{\prime}=0$

Ainsi, la variation relative du débit $Q$ correspondant à un NPSH donné est égal à la variation $\Delta a_{1} / a_{1}$

Dans le cas d'une variation $\Delta \beta_{1}$ de l'angle moyen d'entrée des aubes on obtient :

$$
\left(\frac{\Delta Q}{Q}\right)_{\beta_{1}}=\frac{\cos \beta_{1}}{\sin \beta_{1}-\frac{K^{\prime} Z_{R} \cdot e_{1}}{\pi D_{1}}} \cdot \Delta \beta_{1}\left(\beta_{1} \text { radian }\right)(12)
$$

Les écarts observés sur l'angle moyen $\beta_{1}$ peuvent être considérés comme du même ordre de grandeur que ceux qui affectent $\beta_{2}$. A titre d'exemple une incertitude de $30^{\prime}$ sur un angle moyen $\beta_{1}$ de $16^{\circ}$ pour $Z_{R}=6$, $D_{1}=0,5 \mathrm{~m}, e_{\mathrm{I}}=10 \mathrm{~mm}$ conduirait à une incertitude relative $(\Delta Q / Q)_{\beta_{1}}= \pm 3,5 \%$.

Influence de la variation de l'épaisseur moyenne d'entrée des aubes $e_{T}$

La dérivation de la relation (11) par rapport à $e_{1}$ montre que la variation du débit $Q$ correspondant à un NPSH donné, conduit à l'expression :

$$
\left(\frac{\Delta Q}{Q}\right)_{e_{1}}=\frac{-K^{\prime}}{\frac{\pi D_{1} \cdot \sin \beta_{1}}{Z_{R} \cdot e_{1}}-K^{\prime}} \cdot \frac{\Delta e_{1}}{e_{1}}
$$

Les écarts observés sur $e_{1}$ peuvent être considérés comme du même ordre de grandeur que ceux qui affectent $e_{2}$. A titre d'exemple, une incertitude $\Delta e_{1} / e_{1}= \pm 20 \%$ avec $\beta_{1}=16^{\circ}, Z_{R}=6, D_{1}=0,5 \mathrm{~m}$, $e_{1}=19 \mathrm{~mm}$, conduirait à une incertitude $(\Delta Q / Q)_{e_{1}}$ pouvant dépasser $\pm 3 \%$.

\section{Influence de la variation du jeu aux étanchéités}

Le débit de fuite $q$ dans un jeu cylindrique d'entrefer $j$, de longueur $L$, de diamètre $D$ soumis à une différence de hauteur $\Delta H$, est donné par une relation de la forme :

$$
q=\frac{\pi D j}{\frac{\lambda L}{2 j}+K} \sqrt{2 g \Delta H}
$$

Le débit utile $Q$ subit donc une variation relative à hauteur énergétique totale constante $(\Delta Q / Q)_{j}=-k \Delta q / q \times 1-\eta_{\nu} / \eta_{\nu}$

$\eta_{\nu}=$ rendement volumétrique. 
En dérivant l'expression (14) par rapport à $j$ on trouve :

$$
\left(\frac{\Delta Q}{Q}\right)_{j}=-k \frac{\Delta j}{j} \times \frac{1-\eta_{v}}{\eta_{v}}
$$

$k$ est un facteur de forme qui varie de 1 à 1,5 selon l'importance relative des pertes par frottement et des pertes singulières dans les labyrinthes.

Les caractéristiques hauteur-débit et puissance-débit se trouvent donc translatées en débit de la quantité $(\Delta Q)_{j}$ à hauteur et à puissance constantes. Le rendement, lui, est affecté comme le débit :

$$
\left(\frac{\Delta \eta}{\eta}\right)_{j}=\left(\frac{\Delta Q}{Q}\right)_{j}
$$

L'incertitude sur les jeux peut être imputable aux imprécisions d'usinage ainsi qu'à une variation dans le temps dûe aux usures. Parfois, le bureau d'études est amené à prévoir volontairement des jeux non en similitude sur le modèle réduit et sur la machine industrielle pour des raisons de comportement mécanique.

A titre d'exemple on peut voir qu'une variation $\Delta j / j$ de $50 \%$ pourrait conduire à une variation $(\Delta Q / Q)_{j}$ ou $(\Delta \eta / \eta)_{j}$ de 1 à $2 \%$ environ.

\section{Influences d'autres variations}

C'est volontairement que nous ne ferons que mentionner ici certains paramètres dont la variation peut être significative mais qui nécessiteraient de beaucoup plus longs développements. Il convient de signaler entre autres l'influence des états de surface et du nombre de Reynolds sur le rendement en précisant que pour les pompes d'assez faible vitesse spécifique, l'état de surface de l'organe de récupération devient plus important que celui des canaux de la roue. Il faut aussi relever l'importance de toute variation de la section de contrôle de l'organe de récupération (ouverture du diffuseur, section de sortie de la volute) qui peut décaler de façon très sensible le débit de rendement maximal ainsi que la valeur de ce rendement.

\section{Combinaison des effets des différents écarts de simi- litude}

Dans la pratique, on suppose que la dispersion des différentes cotes géométriques présente un caractère gaussien. Les écarts de similitude correspondant à un niveau de probabilité de $95 \%$ sont donc égaux au double de l'état type de chaque dispersion.

Lorsque les différents écarts géométriques mentionnés ci-dessus $\left(\Delta b_{2}, \Delta \beta_{2}, \Delta e_{2}\right)$ représentent des écarts aléatoires (donc probables), ils peuvent être considérés comme indépendants. On détermine l'incertitude globale $\Delta Q / Q$ en calculant la moyenne quadratique des incertitudes élémentaires $(\Delta Q / Q)_{b_{2}},(\Delta Q / Q)_{\beta_{2}}$ et $(\Delta Q / Q)_{e_{2}}$. Cette incertude globale présente à son tour un niveau de probabilité de $95 \%$.

Il convient évidemment de dissocier des écarts probables $\Delta b_{2}, \Delta \beta_{2}, \Delta e_{2}$ les écarts systématiques connus

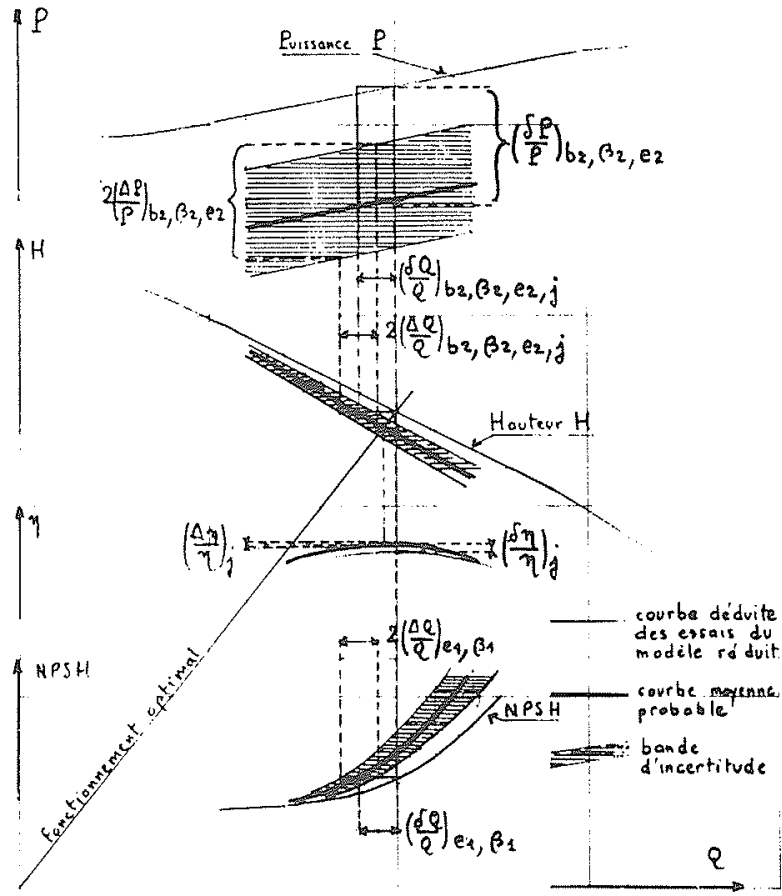

Figure 1 - Etablissement des caractéristiques prévisionnelles

$\delta b_{2}, \delta \beta_{2}, \delta e_{2}$ qui conduisent non pas à une incertitude mais à un déport de caractéristique. Le déport global $\delta Q / Q$ sera alors la somme algébrique des déports élémentaires $(\delta Q / Q)_{b_{2}},(\delta Q / Q)_{\beta_{2}},(\delta Q / Q)_{e_{2}}$

La détermination des caractérisitques prévisionnelles peut se faire comme indiqué sur la figure 1.

Etude des écarts de similitude géométrique relatifs à quelques réalisations industrielles

\section{Etude comparative des résultats d'essais des pompes de circulation de condenseur}

La quasi totalité des pompes de circulation de condenseur utilisées par EDF sont des pompes géométriquement semblables, au rognage près de la roue, construites par le même fabricant sur le même tracé hydraulique. Outre leur taille liée au coefficient de similitude et au rognage de la roue, elles diffèrent par de légères variations de certains paramètres (largeur de sortie, épaisseur des ailettes, etc...).

Or, notamment dans les années soixante, la plupart de ces pompes étaient essayées dans des conditions de mesure généralement bonnes et très souvent étroitement comparables. Ceci a donc été mis à profit pour rassembler et comparer ces résultats d'essais après les avoir mis sous forme de coefficients adimensionnels :

- Coefficient de débit $\varphi=\frac{Q}{U_{2} \cdot \pi D_{2} b_{2}}$

- Coefficient de hauteur $\quad \psi=\frac{2 g H}{U_{2}^{2}}$

- Coefficient de puissance $\chi=\frac{2 P}{\rho U_{2}^{3} \pi D_{2} b_{2}}=\frac{\varphi \psi}{\eta}$ 


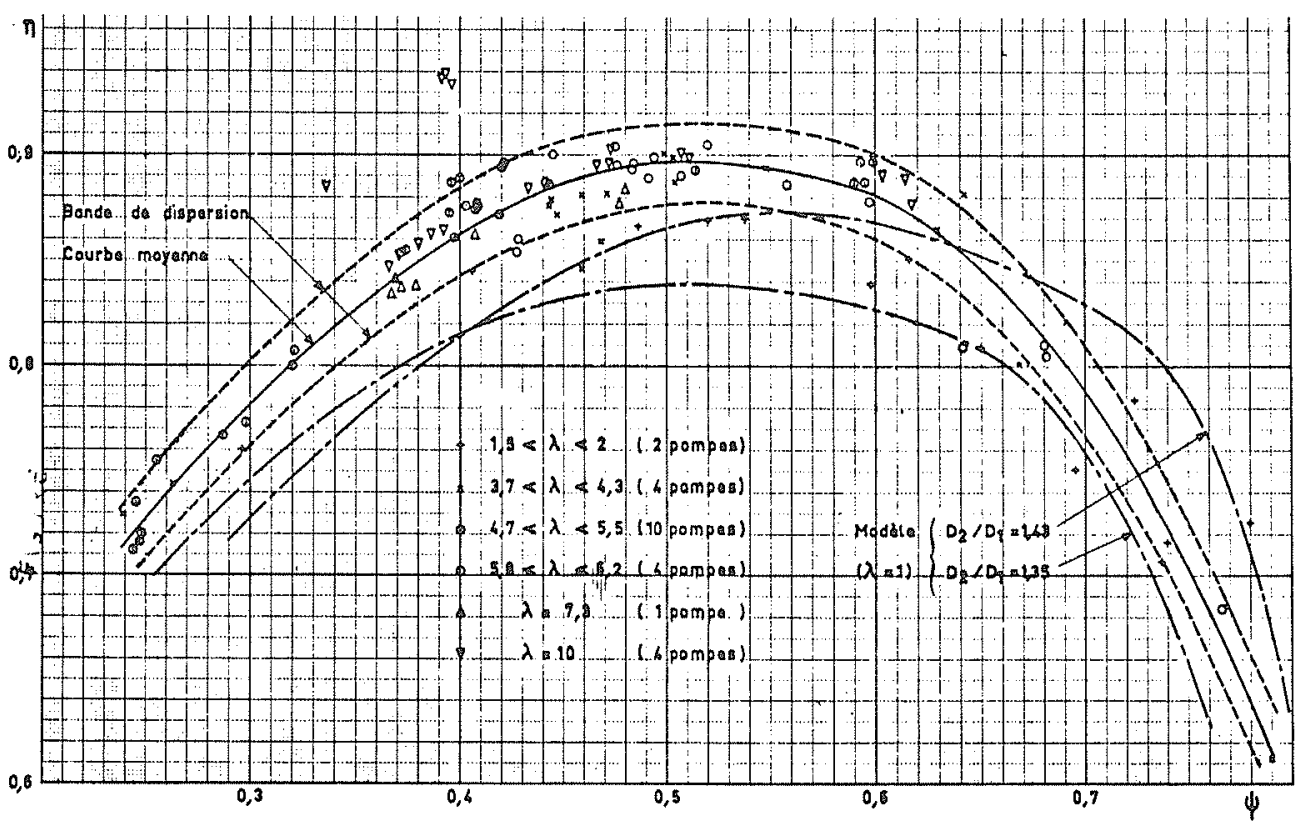

Figure 2 - Rendement-hauteur.

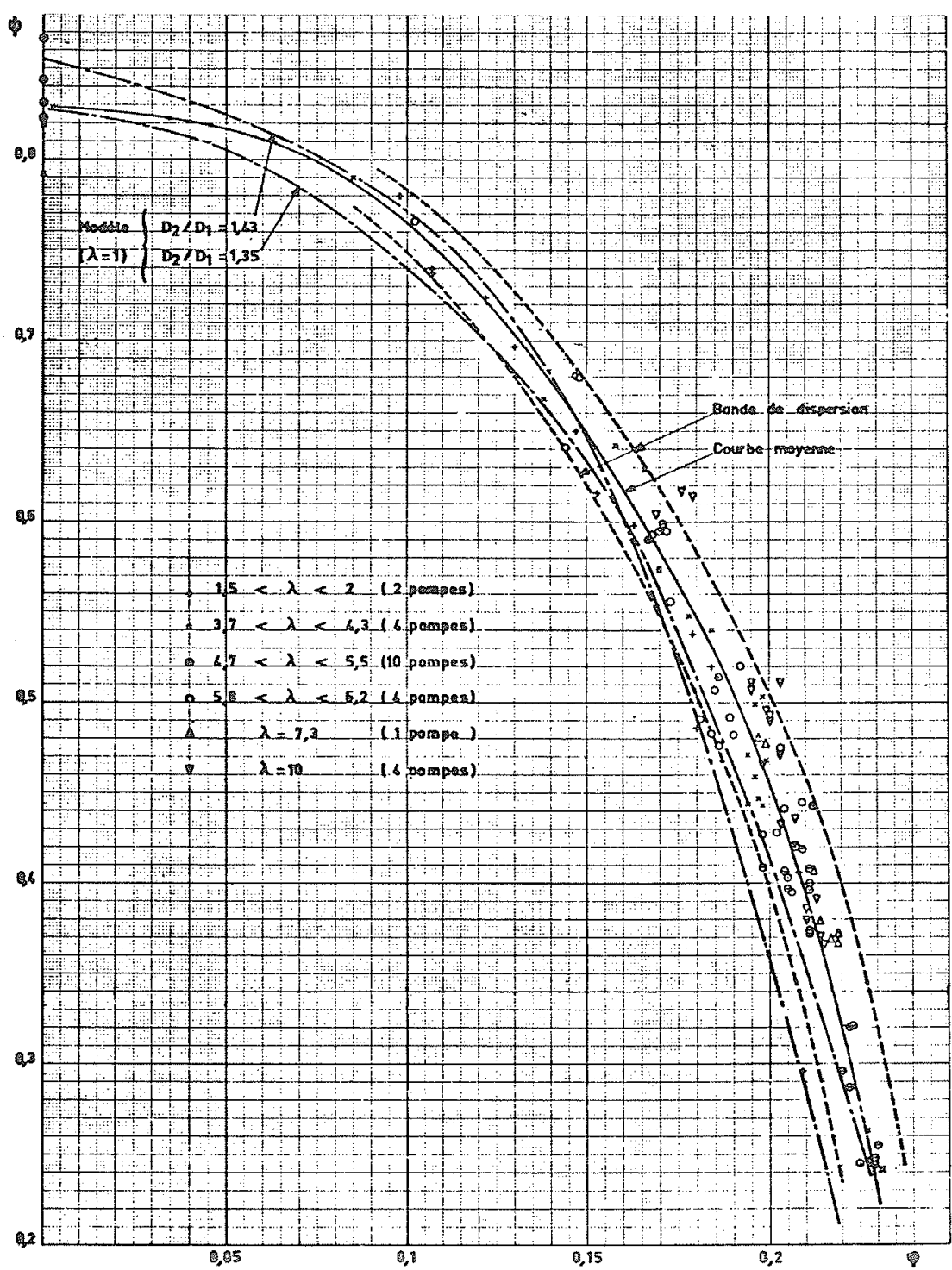

Figure 3 - Hauteur-débit 
où $D_{2}$ et $b_{2}$ sont le diamètre et la largeur de sortie de la roue et $U_{2}$ la vitesse d'entraînement à la sortie.

Les figures 2 et 3 représentent, à titre d'exemple, les graphiques $\psi(\varphi)$ (hauteur-débit) et $\eta(\psi)$ (rendement hauteur). La statistique porte sur 22 pompes dont l'échelle, par rapport au modèle réduit, va de 1,5 à 10 environ. Rappelons qu'il s'agit là de pompes centrifuges à volute dont le coefficient de vitesse angulaire est voisin de 2. $\left(n_{s} \# 100\right)$

Il est évidemment difficile, du fait de la dispersion due aux incertitudes de mesure, de mettre en évidence l'influence séparée des divers écarts de similitude, d'autant plus que nous ne disposons pas toujours de tous les relevés dimensionnels nécessaires. Si on met à part les deux pompes les plus petites, très proches du modèle, on peut toutefois remarquer:

- Que la courbe rendement-hauteur offre peu de dispersion (environ \pm 2 points en rendement, ce qui est du même ordre que les incertitudes de mesure) et ne fait pas clairement apparaître d'effet d'échelle lié à la taille des pompes;

- Ques les points sont plus dispersés sur la courbe hauteur-débit ( $\pm 5 \%$ sur $\varphi$ à $\psi$ donné), ce qui tendrait à confirmer que c'est surtout le débit qui est sensible aux écarts de similitude. Compte tenu d'une valeur de l'erreur limite sur la mesure du débit d'environ $1.5 \%$, il apparait que les autres incertitudes introduisent une dispersion voisine de $\pm 4,5 \%$. Dans cette dispersion, les écarts de similitude interviennent sans doute pour une large part. On peut effet estimer ces écarts à :

$$
\begin{array}{ll}
\frac{\Delta b_{2}}{b_{2}}= \pm 2 \% & \text { d'où } \quad\left(\frac{\Delta Q}{Q}\right)_{b_{2}}= \pm 2 \% \\
\Delta \beta_{2}= \pm 30^{\prime} & \text { d'où } \quad\left(\frac{\Delta Q}{Q}\right)_{\beta_{2}}= \pm 1,5 \% \\
\frac{\Delta e_{2}}{e_{2}}= \pm 15 \% & \text { d'où } \quad\left(\frac{\Delta Q}{Q}\right)_{e_{2}}= \pm 1 \%
\end{array}
$$

Cette étude statistique a été la base d'une méthode largement utilisée par EDF permettant de contrôler les performances d'une pompe de la famille en l'affranchissant de la mesure du débit toujours assez lourde à mettre en œuvre.

\section{Etude du cas des pompes primaires de réacteur PWR}

Les réacteurs à eau pressurisée $900 \mathrm{MWe}$ du programme nucléaire français sont équipés de 3 pompes primaires qui délivrent chacune un débit de $6,3 \mathrm{~m}^{3} / \mathrm{s}$ sous une hauteur de $79 \mathrm{~m}$. La pompe comporte 1 roue d'un étage de type hélicocentrifuge.

Les caractéristiques hydrauliques prévisionnelles de chaque roue sont déterminées par application d'un programme de calcul de similitude à partir d'essais faits sur un modèle réduit, en prenant en compte les écarts dimensionnels de réalisation des pièces. Pour cela un relevé dimensionnel d'un certain nombre de grandeurs (diamètre de sortie $D_{2}$, largeur de sortie $b_{2}$, ouverture de de sortie $a_{2}$, épaisseur de sortie $e_{2}$ ) est effectué sur chacune des roues industrielles, ainsi que sur le modèle ré- duit. Les résultats de ces mesures sont introduits dans le programme de calcul des caractéristiques hydrauliques pour corriger les lois de similitude, de façon similaire à ce qui a été indiqué au chapitre précédent, avec l'emploi des formules simplifiées. La validité du programme de calcul a été contrôlée par des essais hydrauliques à plein débit effectués sur la première pompe de la série.

L'importance du programme nucléaire français de réacteurs $900 \mathrm{MWe}$ en cours de réalisation permet de disposer d'un nombre élevé de pompes identiques de grande puissance. A partir des relevés dimensionnels mentionnés précédemment, une étude statistique des écarts géométriques de réalisation à été entreprise. Cette étude porte sur 40 roues provenant de 4 foumisseurs différents (repères $A, B, C, D$ ). Elle nous permet ainsi d'avoir une assez bonne idée :

- en premier lieu des écarts de réalisation auxquels on peut s'attendre entre les roues d'une série (7 à 15 pièces) coulées d'après un même modèle de fonderie ;

- par ailleurs, des écarts qui peuvent exister entre des séries de roues exécutées à partir de modèles de fonderie différents mais issus d'un même plan de bureau d'études.

La figure 4 montre la dispersion obtenue sur $b_{2}$, $\beta_{2}$ et $e_{2}$. On peut noter que les réalisations des différents fcurnisseurs ont des écarts types de dispersion variant dans un rapport de 1 à 3 ; par exemple sur $b_{2}$, l'écart type varie de $0,5 \%$ à $1,31 \%$ suivant les fournisseurs. Il s'ensuit que l'écart type global résultant est augmenté de façon sensible et que la courbe de dispersion s'éloigne de l'allule gaussienne.

Les résultats de l'étude statistique donnent pour l'ensemble des roues considérées et pour un niveau de
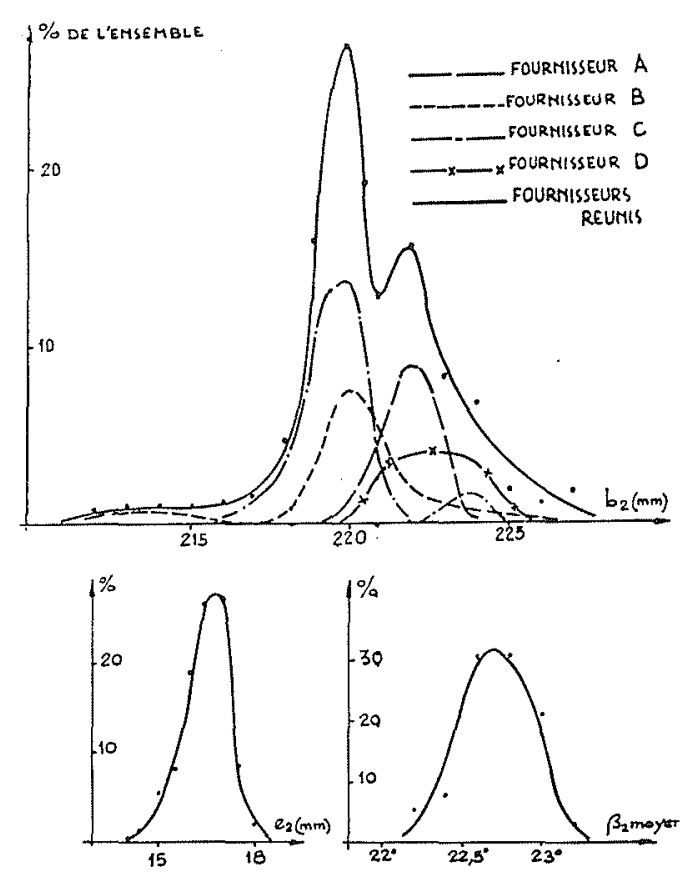

Figure 4 
probabilité de $95 \%$ les incertitudes suivantes:

$$
\begin{array}{ll}
\operatorname{sur} b_{2} & \Delta b_{2} / b_{2}= \pm 2,15 \% \\
\operatorname{sur} e_{2} & \Delta e_{2} / e_{2}= \pm 10,5 \% \\
\operatorname{sur} \beta_{2} & \Delta \beta_{2} / \beta_{2}= \pm 2 \%
\end{array}
$$

L'application des formules exposées au chapitre précédent conduit, pour le point nominal, aux écarts de caractéristique probables suivants imputables aux seuls aléas de fabrication :

$$
\begin{array}{ll}
\Delta Q / Q= \pm 3,5 \% & \text { à la hauteur nominale } \\
\Delta H / H= \pm 8,5 \% & \text { au débit nominal }
\end{array}
$$

Ces incertitudes sur les caractéristiques apparaissent incompatibles avec le domaine de fonctionnement assurant un bon comportement du cœur du réacteur. Ceci justifie donc pleinement la nécessité d'un relevé dimensionnel précis de chaque roue et l'utilisation d'un programme de correction des cotes théoriques du tracé hydraulique.

Si la caractéristique prévisionnelle ainsi déterminée ne passe pas dans le domaine de tolérance admis, la roue est retouchée de deux manières : soit par rognage dans le cas d'une roue surpuissante, soit par affûtage intrados (sur l'arrière) de la sortie des aubes dans le cas contraire.

Comme déjà signalé dans l'introduction, il faut bien voir qu'après ces corrections, il subsistera encore sur les courbes de caractéristiques prévisionnelles une incertitude provenant à la fois :

- des erreurs de mesures hydrauliques lors des essais modèle réduit

- des effets d'échelle entre modèle réduit et machine industrielle

- des erreurs de mesures dimensionnelles tant sur le modèle que sur la roue échelle 1 .

Les incertitudes sur les caractéristiques imputables à ce dernier point peuvent être évaluées à

$$
\begin{array}{ll}
\Delta Q / Q= \pm 0,6 \% & \text { à la hauteur nominale } \\
\Delta H / H= \pm 1,4 \% & \text { au débit nominal }
\end{array}
$$

\section{Etude du cas de turbines-pompes}

Le problème qui se pose dans le cas des turbines- pompes peut se résumer ainsi :

A partir d'un tracé théorique parfaitement défini, on met au point un modèle réduit donnant des performances hydrauliques satisfaisantes. Il s'agit ensuite de définir le tracé théorique de la machine industrielle en tenant compte des écarts inévitables et parfois volontaires entre modèle réduit et tracé théorique initial ainsi que des aléas de construction imposés par les techniques de fabrication. Il en résulte deux nécessités essentielles pour obtenir à coup sûr des caractéristiques hydrauliques industrielles à l'intérieur de la fourchette de garantie :

- un contrôle aussi précis que possible des formes et des écarts géométriques ;

- une quantification des écarts hydrauliques résultants.

Nous présentons dans ce qui suit le cas particulier des turbines pompes multicellulaires de La Coche.

Seules les formes gauches des aubes de roues et des ailettes des canaux de retour semblent être sources d'écarts géométriques notables, et ce sont aussi les surfaces qui ont le plus d'influence sur les caractéristiques hydrauliques. Il a été procédé à des relevés dimensionnels aube par aube des roues du modèle réduit et des machines industrielles. Les figures 5 et 6 montrent les écarts observés sur l'angle de sortie $\beta_{2}$. L'intervalle d'appréciation de $0,2^{\circ}\left(12^{\prime}\right)$ apparaît pour l'instant comme un minimum réalisable.

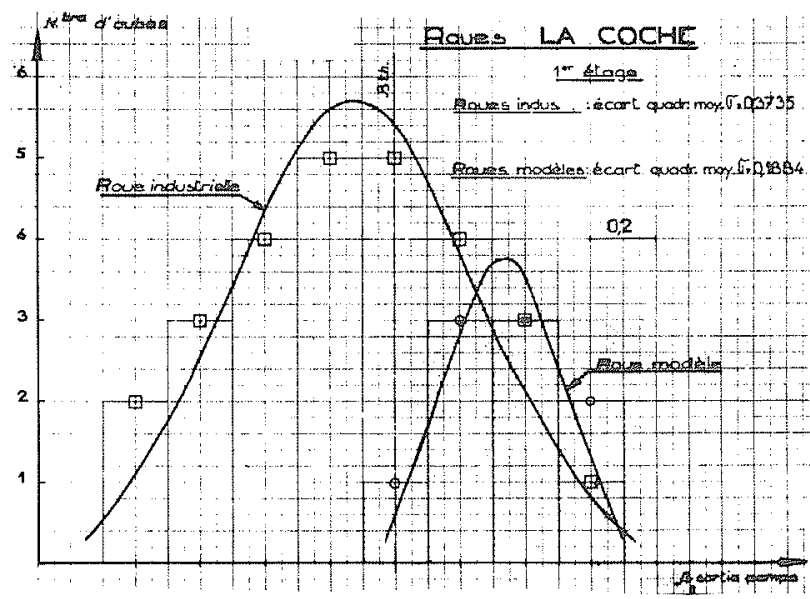

Figure 5

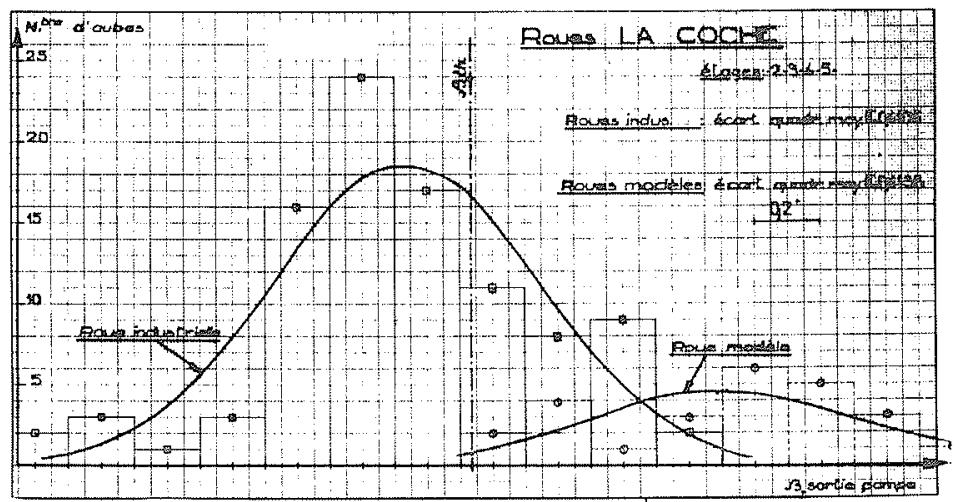


On constate les écarts moyens suivants :

$\begin{array}{ll}\Delta \beta_{2}=+0,5^{\circ} & \begin{array}{l}\text { entre modèle réduit et plan de tracé } \\ \text { théorique }\end{array} \\ \Delta \beta_{2}=-0,3^{\circ} & \begin{array}{l}\text { entre machine industrielle et plan de } \\ \text { tracé théorique }\end{array}\end{array}$

On a observé par ailleurs sur la largeur de sortie $b_{2}$, des écarts négligeables entre le modèle réduit, les machines industrielles et le plan de tracé théorique. En contre partie les écarts d'épaisseur $\Delta e_{2} / e_{2}$ ont été en moyenne de $+10 \%$ en passant du modèle réduit aux machines industrielles.

Dans le cas particulier de La Coche, on a été conduit aux conclusions suivantes :

- Les formules simplifiées du chapitre précédent semblent tout à fait satisfaisantes pour expliquer l'ordre de grandeur des écarts de caractéristiques constatés entre le modèle réduit et les machines industrielles.

- L'incertitude sur $\beta_{2}$ explique l'essentiel des écarts hydrauliques constatés. Il convient de noter que le contrôle de $\beta_{2}$ est très délicat car il faut apprécier des écarts inférieurs à $0,3^{\circ}$. La taille des modèles réduits rend ces contrôles particulièrement difficiles car un écart de $0,5^{\circ}$ sur une longueur d'aube de $100 \mathrm{~mm}$ ne conduit qu'à un écart géométrique de $0,87 \mathrm{~mm}$. On peut dire finalement qu'au prix de contrôles géométriques très serrés, donc onéreux, on peut estimer assez bien l'ordre de grandeur des écarts hydrauliques provenant des aléas de fabrication au voisinage du rendement maximal, notamment sur la caractéristique hauteurdébit.

Valeur garantie et tolérance contractuelle. Valeur prévisionnelle, incertitude probabilisée de construction de la pompe et incertitude probabilisée de conception du circuit

Ce sont d'abord les exigences du client, imposées au contrat sous forme de tolérances sur les caractéristiques demandées, qui déterminent, dans l'action du foumisseur, la classe d'exécution et l'estimation des aléas.

Une tolérance acceptée par le "foumisseur-constructeur" doit être au moins égale à l"incertitude" globale du constructeur qui comprend les incertitudes de la construction et les "incertitudes" couvrant les aléas propres à un contrat donné.

Cette tolérance, acceptée par le "client-concepteur" doit être compatible avec les nécessités du procédé industriel dans lequel s'intègre l'opération de pompage. A l'incertitude de conception qui en résulte doivent être ajoutées les "incertitudes" couvrant les aléas du contrat, estimées cette fois-ci par le client.

Cette tolérance ainsi déterminée, qui accompagne une valeur garantie au contrat, constitue un compromis entre les marges nécessaires pour une fabrication donnée et les marges acceptables pour une utilisation donnée. Une valeur garantie peut donc être assez éloignée de la valeur prévisionnelle correspondante, c'est-à-dire la valeur pourvue d'une incertitude probabilisêe de construction ou d'une incertitude probabilisée de conception.

Elle peut être d'autant plus éloignée que, souvent, les incertitudes de mesure sont incorporées dans cette garantie, après avoir été majorées pour "éviter toute discussion". Notons que cette façon de faire est parfois à reconsidérer.

Il n'est pas toujours facile de déterminer les incertitudes de conception admissibles pour un circuit de pompage. Le concepteur fixera alors son choix en fonction de tolérances telles que celles proposées dans certains codes d'essai.

Mais lorsque l'importance des risques techniques ou des conséquences financières le nécessite, le concepteur peut avoir des exigences "sur mesure" : alors, il estime le débit minimal requis dans plusieurs situations de fonctionnement. Il estime également le débit maximal admissible à 1" "égard des sollicitations hydro-mécaniques, de la puissance consommée et du dimensionnement de l'installation". Enfin, il fixe son débit prévisionnel, c'est-à-dire le débit le plus probable qu'il désire. Le constructeur doit faire de cette valeur sa valeur prévisionnelle et devrait foumir au concepteur du circuit l'incertitude de construction. Ce renseignement est en effet d'un grand intérêt dans le cas de pompes faisant l'objet d'une optimisation soignée; il est préférable de se baser sur une valeur prévisionnelle plutôt que sur une valeur garantie. Il y a lieu toutefois de vérifier les conséquences d'une valeur obtenue qui se situerait à la limite de la garantie ou d'estimer les possibilités de correction de cette valeur.

Autour de la valeur prévisionnelle, on examine l'effet global sur le fonctionnement et le dimensionnement que prođuit la différence simulée. Avec des données ainsi plus précises, on évite un surdimensionnement en chaîne des matériels.

Cette façon de faire vaut surtout d'être utilisée quand la pompe concernée est une machine principale du système de production, c'est-à-dire une pompe pour laquelle un mauvais dimensionnement entraîne soit une perte de production, soit un surinvestissement de l'installation. Si l'effet sur la production d'une différence de débit est assez facile à mettre en évidence, l'effet sur le surdimensionnement du circuit est plus difficile à établir : chaque appareil possède une capacité d'absorption des marges par laquelle une certaine variation du débit reste sans influence sur le dimensionnement de cet appareil.

Parallèlement à la recherche de ces effets, le concepteur du circuit doit s'efforcer de contrôler et régler, au cours du projet, les pertes de charge du circuit. Elles sont souvent une cause prépondérante des écarts observés sur le débit. Il peut être souhaitable dans certains cas, de réaliser une maquette.

Une meilleure précision d'un débit résultant d'une caractéristique de pompe et d'une caractéristique de circuit dépend donc largement du constructeur de la pompe et du concepteur du circuit. Mais elle dépend aussi de la précision de la mesure qui peut être de $0,5 \%$ dans de bonnes conditions de mesure mais peut atteindre 2 à $3 \%$ dans certains cas. 
Exemple : Pompes de circulation du réfrigérant primaire d'un réacteur nucléaire (Fessenheim 1 et 2$)\left({ }^{1}\right)$

- Débit prévisionnel ("best estimate") $Q p$

- Débit de conception thermohydraulique $Q p-3,2 \%$ (minimal admissible)

- Débit de dimensionnement mécanique $Q p+4,2 \%$ (maximal admissible)

(1) D'après note EDF-SEPTEN E-SE/PI 79-03 - PE 79-25
- Incertitude de mesure

- Débit moyen mesuré sur 3 pompes ( $\mathrm{y}$ compris incertitudes de mesure).

- Fessenheim 1

$+2,2 \%$

$-2 \%$

- Fessenheim 2

$+1,2 \%$

$Q p$

\section{Discussion}

Président : M. P. BERGERON

M. le Président. - Vous avez constaté que la teneur de cette communication ne pouvait laisser indifférents aussi bien les Constructeurs que les Utilisateurs. Y a-t-il des interventions ?

M. FANELLI. - La communication de M. CANAVELIS m'a particulièrement intéressé. En Italie, en effet, dans le cadre du Comité Technique CT4 du CEI, on a essayé de faire une analyse rationnelle de certains des aléas qui viennent d'être mis en évidence. Il est bien évident qu'il y a des risques pour les deux parties du contrat : le "risque du constructeur", c'est-à-dire le risque de se voir refuser, sur la base des essais de réception, une machine qui en réalité est bonne ; et le "risque du client", c'està-dire le risque d'accepter sur la base des essais de réception une machine qui en réalité ne répond pas aux garanties. Si les tolérances sont fixées par un compromis "quelconque", c'est-à-dire non rationnel, ces risques restent dans l'ombre et peuvent être non balancés d'une façon équitable entre le constructeur et le client. Il est par contre possible de formuler une analyse statistique quantitative de ces risques, de façon à éclaircir les conséquences économiques et réaliser le compromis ci-dessus d'une façon équitable. Une telle étude, dont une ébauche a été proposée par le Comité CT4 italien au CEI., devrait s'insérer dans le dialogue préconisé par M. CANAVELIS entre constructeurs et utilisateurs et tenir compte, bien entendu, des précisions apportées par la contribution de M. CANAVELIS sur les aléas que supporte le constructeur.

Evidemment, ces considérations statistiques ont un sens "en moyenne", sur un grand nombre de machines plus ou moins homogènes, et un sens beaucoup moins précis pour une seule machine ou pour un petit nombre de machines. Pour un grand constructeur, il n'en est pas moins vrai que ces considérations pourraient l'aider à ajuster son propre risque d'une façon équitable en moyenne.

M. GILMER. - Je voudrais insister sur un point : il arrive souvent et notamment pour les pompes nucléaires, qu'on soit amené à garantir trois points de la courbe ; si le point nominal est rela tivement facile à obtenir, il est très difficile d'approcher le point à vanne fermée et le point situé très à droite de la caractéristique par des affûtages des ailes à l'entrée et à la sortie pour compenser les aléas de fonderie.

Il est certain que, pour les turbines-pompes, le problème est très différent; les roues sont usinées sur toute leur surface et les résultats sont moins aléatoires. Par contre, pour les pompes multicellulaires comme celles de La Coche, le problème réapparaît avec les roues moulées.

Une des difficultés n'a pas été prise en compte dans l'exposé de M. CANAVELIS : le décalage des noyaux à la fonderie. Il a été tenu compte des écarts d'épaisseurs dûs aux décalages dans un même plan radial. Les décalages axiaux aléatoires des noyaux sont aussi importants.
Dernier point, le code est un carcan où il est imposé des tolérances très faibles pour l'obtention des caractéristiques, que ce soit pour de grandes roues ( 1 ou plusieurs mètres) ou pour de petites roues $(10 \mathrm{a} 30 \mathrm{~cm})$. Il semble que l'on ait oublié la notion de dimensions. Le même code est utilisé pour des pompes de $10 \mathrm{~kW}$ et des pompes de plusieurs milliers de $\mathrm{kW}$. C'est un nonsens. Il arrive parfois que les constructeurs soient obligés de dépenser plus que la valeur de la pompe pour la faire accepter; c'est aberrant.

M. CANAVELIS. - J'ai fait allusion, dans mon exposé, aux codes d'essai de pompes ISO qui sont couramment employés pour les essais de pompes industrielles courantes. Il convient de citer également l'existence d'autres codes d'essai tels le code EUROPOMPES ou les codes CEI., chaque code étant plus particulièrement adapté à un type de pompe.

M. GUITON. - M. CANAVELIS et ses co-auteurs viennent de nous montrer à quels déplacements de la caractéristique hauteur-débit on devait s'attendre, compte tenu des écarts géométriques existants entre des pompes réputées semblables. Leur analyse se limite à la zone de rendement maximal.

C'est cependant le point de départ obligatoire et la base de référence d'une étude plus complète à effectuer s'étendant jusqu'au fonctionnement à débit nul des pompes.

Dans bien des cas en effet, la plage de variation des courbes hauteur-débit et puissance-débit va en augmentant au voisinage du débit nul.

Les limitations parfois imposées dans les contrats sur la hauteur maximale à débit nul à ne pas dépasser par suite de la pression de timbre de l'installation,ou de la puissance à débit nul à ne pas dépasser par sulte des possibilités du moteur d'entrainement, imposent de mieux connaître cette plage d'incertitude.

Dans le cadre du Groupe de Travail $\mathrm{n}^{\circ} 1$ de la Division "Applications Industrielles de la Mécanique des Fluides" de la SHF., le Sous-groupe de Travail 1c, qui s'occupe de 1'"Influence des divers paramètres géométriques et hydrauliques sur les courbes $\bar{H}-\bar{Q}$ et $\bar{P}-\bar{Q} \dot{a}$ débit partiel des pompes compresseurs et ventilateurs" serait donc heureux si, en partant de ce point de départ, une communication au moins pouvait être présentée sur ce sujet lors d'une prochaine Session du Comité Technique.

La Société HYDROART lui a déjà fourni un premier document s'y rapportant. Le sous-groupe de travail $1 c$ l'en remercie.

M. CASACCI. - La précision des formes géométriques des tracés et leur influence sur les caractéristiques hydraúliques des turbomachines hydrauliques est une question importante qui devrait intéresser autant 1'utilisateur que le constructeur. On doit remercier M. CANAVELIS d'avoir fait une première analyse de ce sujet.

La définition des erreurs possibles est chose délicate car les écarts d'épaisseur et d'angles peuvent être positifs ou négatifs. 
M. CANAVELIS. - Dans les exemples cités, les angles sont venus de fonderie trop fort ou trop faibles sur toutes les aubes d'une même roue. Peut-on imaginer des cas où l'erreur a été la même sur toutes les aubes d'une même roue?

M. CASACCI. - C'est difficile à dire. Nous nous proposons de faire une étude un peu plus précise. Nous avons eu la chance d'avoir la commande de Grand Maison ; nous aurons à foumir 32 roues plus les rechanges, ce qui constitue un lot assez important. On pourra peut-être en tirer des données statistiques.

Vous avez surtout analysé l'influence de l'entrée et de la sortie, facteurs effectivement importants, il reste cependant entre l'entrée et la sortie une bonne longueur d'aube. $M$. GILMER a fait allusion au déplacement de noyaux. Nous avons constaté sur des grosses roues des déplacements de noyaux très importants qui augmentaient la largeur d'un canal et réduisaient celle du conduit contigu. L'influence de ces défauts ne doit pas être négligeable.

Pour les grosses turbines hydrauliques du type Francis, la fabrication par éléments séparés assemblés par soudure permet d'obtenir une excellente précision géométrique des tracés. Pour une roue de $7,50 \mathrm{~m}$ de diamètre, les défauts de positionnement d'une aube par rapport à l'autre restent de l'ordre de $\pm 2 \mathrm{~mm}$. Sur les roues des turbines-pompes, moulées, on contrôle assez bien les formes d'entrée et de sortie, il est beaucoup plus difficile de s'assurer de la conformité géométrique du reste de l'aube. On pourrait envisager des roues mécanosoudées, mais elles cô̂teraient sensiblement plus cher. Le client accepterat-il de payer un supplément de prix pour obtenir une plus grande sécurité des caractéristiques?

M. GAUBAUDAN remarque que l'apport de M. CANAVELIS va dans le sens souhaité par Electricité de France, à savoir une meilleure connaissance objective de sources d'incertitude par concertation entre constructeurs et utilisateurs. Cependant, il souligne la grande différence entre le code ISO qui intéresse des séries de machines et prend en compte les incertitudes de fabrication et le code CEI qui, au moins pour les turbines, les ignore. Il ajoute que, dans l'élaboration des codes, certains constructeurs, poussés par des considérations commerciales, signent des contrats avec des tolérances plus réduites que celles correspondant aux incertitudes de mesure (avec une probabilité raisonnable). Leur action va à l'encontre d'une codification objective.

M. GUITON. - Ce que vous dites est vrai pour les codes turbines, mais par pour les codes pompes, où il y a une fourchette volante sur le débit garanti de $\pm 10 \%$ (il faut se mettre d'accord au départ), qui tient compte des aléas de construction. Le pompier n'a pas la possibilité, comme le turbinier, d'ajuster le distributeur. Pour les pompes, il y a une tolérance sur le débit.

M. BOUSSUGES . - J'ai deux remarques à faire sur l'exposé de M. CANAVELIS en ce qui concerne le problème des imprécisions.

Il a exposé, au début, les imprécisions correspondant à la hauteur de la roue et à l'épaisseur, en prenant des exemples d'écarts. En réalité, les écarts cumulent à la fois les imprécisions de fonderie et les imprécisions de mesures. Pour les deux premiers exemples, les imprécisions sont relativement réduites, tandis qu'il faut noter que, dans les écarts du $\beta_{2}$, l'imprécision de mesure est très importante ; elle est très différente de celle correspondant aux deux mesures précédentes. Je trouve que vous avez été très optimiste quand vous avez situé l'amplitude complète dans $1 / 2^{\circ}$ d'écart. Je me demande si l'on peut parvenir à trouver un appareil qui donne ce calcul; ce n'est pas tellement facile.

Deuxième remarque. M. GILMER, dans son intervention, a dit que c'est relativement plus facile pour les grandes roues de turbines-pompes.

Le Cahier des Charges est en particulier très exigeant concernant la puissance de démarrage en régime normal en pompe. Il ne faut pas oublier que la puissance à débit nul, comme le faisait remarquer M. BERGERON, est beaucoup plus grande quand on s'éloigne du point de fonctionnement nominal. Le
Cahier des Charges nous coince aussi énormément en ce qui concerne les pressions maximales quand la conduite est "tangente" au point de vue surpression.

M. BORCLANL. - D'abord je remercie M. CANAVELIS pour $m$ 'avoir permis de lire le texte de son intervention, en me le donnant à l'avance.

Il me semble qu'on peut faire quelques observations : avant tout il vaut la peine de souligner que le problème des garanties de débit est un problème qui concerne surtout les constructeurs de pompes, c'est-à-dire les constructeurs qui ont à faire avec des machines non munies d'un organe de régulation du débit. En effet le problème perd une bonne partie de son importance lorsque, comme dans le cas des turbines, la garantie concerne une grandeur, la puissance maximale délivrée, qui dépend de l'ouverture du distributeur et donc il n'y a pas de problèmes particuliers sauf celui de prévoir des marges suffisants pendant l'exécution du projet. Ces marges, entre autres, sont favorisés par les mêmes codes CEI, qui permettent de choisir comme puissance de référence pour la comparaison des rendements mesurés et garantis, une puissance égale ou même supérieure à celle établie par le contrat, même si entre certaines limites (10\%).

Ce que M. CANAVELIS a exposé dans son intervention, qui a touché quelques aspects fondamentaux de la recherche de labo. ratoire, même d'autre part à la conclusion suivante : l'étude de l'effet d'échelle doit être effectuée dans les laboratoires, c'est-àdire en opérant sur le même modèle et en modifiant la chute et par conséquent le nombre de Reynolds. En effet les raisons d'incertitude quand on compare les mesures faites sur modèle et celles faites sur la machine industrielle sont tellement nombreuses que l'effet d'échelle - s'il y en a - est pour ainsi dire caché par les imprécisions de mesure et par les inévitables écarts de similitude.

M. CANAVELIS a fait allusion à l'influence de l'angle de sortie en pompe des aubes de la roue sur le débit et à celle analogue de l'angle d'entrée en pompe : j'aimerais savoir si on a fait des observations expérimentales précises sur ce dernier fait.

Enfin je voudrais savoir ce qu'on entend quand on parle d'écarts géométriques systématiques imposés par des procédés de fabrication ou par des exigences mécaniques et qu'on cite les épaisseurs des aubes, A ce propos,je dois remarquer que pour notre part, on a toujours essayé de respecter la similitude entre modèle et machine industrielle sur toutes les êpaisseurs des aubes, soient-elles de roue, de directrice ou d'avant-directrice.

M. CANAVELIS. - Il convient de préciser que dans votre exposé, les angles d'entrée $\left(\beta_{1}\right)$ sont supposés influencer essentiellement la branche à grand débit des courbes NPSH-débit et pratiquement pas les courbes hauteur-débit (sauf sur les pompes de grande vitesse spécifique qui sortent du cadre des roues centrifuges, ici, concernées).

M. BORCLANI. - En ce qui concerne la géométrie, vous avez écrit qu'il y a des écarts systématiques qui correspondent à des écarts connus en moyenne à l'avance et qui sont imposés par des procédés de fabrication ou des exigences mécaniques. Vous y incluez l'épaisseur de base. En général, on cherche à reproduire l'épaisseur de base en suivant les règles, c'est-à-dire avec les tolérances.

M. CANAVELIS. - Cela dépend des procédés de fabrication du modèle réduit. Les fabricants de modèles en plastique parviennent peut-être plus facilement à faire des modèles réduits dont les épaisseurs sont en similitude avec l'épaisseur de la grande machine. Mais il y a des constructeurs - et nous en faisons partie - qui utilisent davantage des modèles réduits venant de fonderie ; la fonderie ne permet pas toujours d'avoir sur le modèle réduit des aubes dix fois moins épaisses que les aubes de la grande machine, lorsque le coefficient de similitude est 10 .

A titre d'exemple, je peux vous dire que, pour une grande machine qui aurait des aubes de l'ordre de $25 \mathrm{~mm}$ d'épaisseur, nous ne pouvons pas demander à un fondeur de faire un modèle réduit de $400 \mathrm{~mm}$ de diamètre avec $2,5 \mathrm{~mm}$ d'épaisseur. On ne fournira jamais, en bronze ou en fonte, une roue qui fait $2,5 \mathrm{~mm}$ d'épaisseur ; elle en aura 3 ou 4,5. 